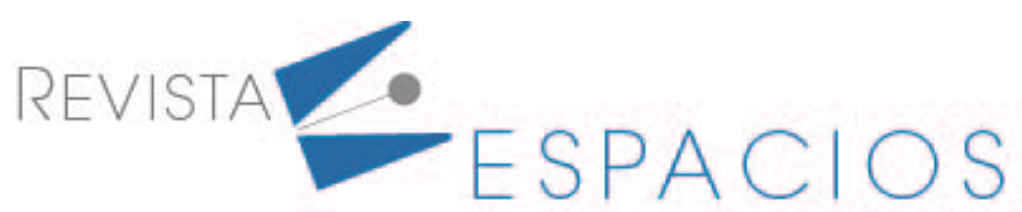

\title{
Signos y síntomas del Síndrome Inflamatorio Multisistémico relacionado con COVID-19 que afecta a niños y adolescentes menores de 19 años en Ecuador
}

\section{Signs and symptoms of multisystem inflammatory syndrome related to COVID-19 that affects children and adolescents under 19 years of age in Ecuador.}

\author{
GARCIA ALVAREZ, Vianca D. ${ }^{1}$ \\ ZUMBA VERA, Dayana P. ${ }^{2}$ \\ NAULA ARCOS, Nicole E. ${ }^{3}$ \\ MONTERO GALARZA, Gloria I. ${ }^{4}$ \\ ZAMBRANO GARCES, Rosa M. ${ }^{5}$
}

\begin{abstract}
Resumen
El Síndrome Inflamatorio Multisistémico (SIM) es una enfermedad que puede desarrollarse tanto en niños como adolescentes menores de 19 años de edad. Esta investigación de enfoque cualitativo y alcance exploratorio describe los signos y síntomas, medios de contagio y los tipos de pruebas que se realizan para el diagnóstico de la misma, y los casos presentados en Distrito de Salud 03D03 del Cantón La Troncal. El reconocimiento temprano del SIM, permite proporcionar un seguimiento contiguo y el tratamiento óptimo según los signos y síntomas que presenten los pacientes.

Palabras clave: COVID-19, SIM pediátrico, Kawasaki, análisis clínico
\end{abstract}

\begin{abstract}
Multisystemic inflammatory syndrome (MIS-C) is a disease that can develop in both children and adolescents under 19 years of age. This research with a qualitative approach and exploratory scope describe the signs and symptoms, means of contagion, and the types of tests that are carried out for its diagnosis, and the cases presented in the 03D03 Health District of the Cantón la Troncal. The early recognition of MIS-C allows providing a contiguous follow-up and the optimal treatment according to the signs and symptoms that the patients present.

Key words: COVID-19, MIS-C pediatric, Kawasaki, clinical analysis
\end{abstract}

\footnotetext{
${ }^{1}$ Estudiante. Ciencias de Salud. Universidad Catolica de Cuenca. Ecuador. viancaleslie04@gmail.com

${ }^{2}$ Estudiante. Ciencias de Salud. Universidad Catolica de Cuenca. Ecuador. johanorla11@gmail.com

${ }^{3}$ Estudiante. Ciencias de Salud. Universidad Catolica de Cuenca. Ecuador.nicol.naula25@gmail.com

${ }^{4}$ Catedratico. Ciencias de Salud. Universidad Catolica de Cuenca. Ecuador gloria.montero@ucacue.edu.ec

${ }^{5}$ Catedratico. Ciencias de Salud. Universidad Catolica de Cuenca. Ecuador rmzambranog@ucacue.edu.ec
} 


\section{Introducción}

La Organización Mundial de la Salud el 15 de mayo de 2020 alertó sobre el Síndrome Inflamatorio Multisistémico que afecta a niños y adolescentes, transtorno con características similares a la enfermedad de Kawasaki y al síndrome de choque tóxico.

El Centro para el Control y Prevención de Enfermedades (CDC, 2020a) indica que esta es una afección en la cual las diferentes partes del cuerpo se pueden llegar a inflamar, incluido el corazón, los pulmones, los riñones, el cerebro, la piel, los ojos u órganos gastrointestinales. Algunos niños desarrollan esta afección después de enfermar por COVID-19 o después de mantener contacto con alguien con el virus, mientras que otros no, por ello es una complicación poco común pero grave, que puede estar relacionada con SARS-CoV-2.

En Ecuador se han notificado algunos casos de niños con este síndrome, por ello el Ministerio de Salud Pública, el 31 de mayo de 2020, realizó una alerta epidemiológica para fortalecer la vigilancia de salud pública y dar seguimiento a los casos reportados. (MSP, 2020a)

Esta investigación establece los diferentes signos y síntomas que presentan los niños y adolescentes que desarrollan síndrome inflamatorio multisistémico; determina el medio de contagio, así como el tipo de pruebas que se realizan para detectar esta afección y el número de casos registrados en el Cantón La Troncal en Ecuador. Para ello, se combina evidencia de los diferentes registros de pacientes que se han atendido, tantos físicos como digitales, durante el período de marzo-agosto de 2021, registros estos almacenados en los repositorios del Distrito de Salud 03D03 del Cantón.

\section{1. ¿Qué es el Síndrome Inflamatorio Multisistémico (SIM)?}

El Síndrome Inflamatorio Multisistémico es una enfermedad grave que ocasiona que algunas partes del cuerpo se inflamen, y afecta a niños y adolescentes. Esta afección tiene similitudes con la enfermedad de Kawasaki y el síndrome del choque tóxico debido a que presenta algunos de los mismos síntomas. (Healthy Children, 2020)

\section{2. ¿Cuáles son los síntomas del síndrome inflamatorio multisistémico?}

Según un informe del Centro para el control y la prevención de enfermedades (CDC, 2020b) el SIM se manifiesta con los siguientes síntomas:

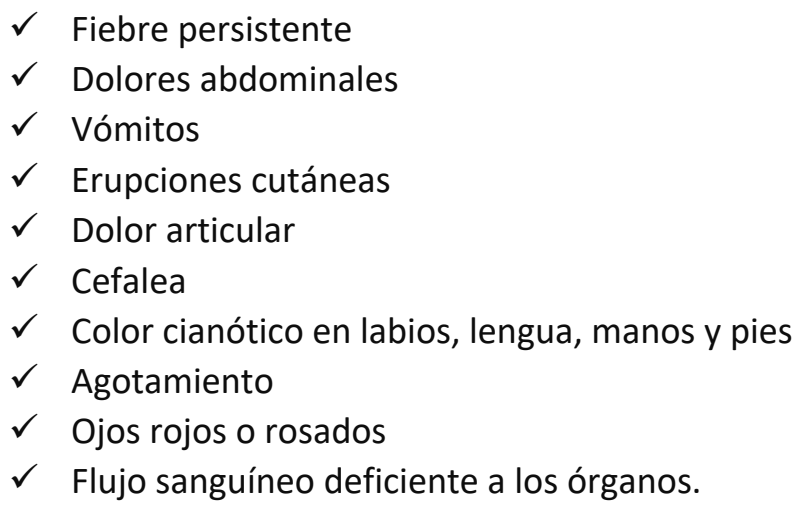

También indica que no todos los niños tendrán los mismos síntomas, por lo que podrían presentar algunos signos preocupantes como los que se detallan a continuación:
$\checkmark$ Dificultad respiratoria
$\checkmark$ Dolor o presión en el pecho persistente
$\checkmark$ Confusión 
$\checkmark$ Incapacidad de despertarse o permanecer despierto.

\subsection{Diagnóstico}

La Sociedad Chilena de Infectología (SOCHINF, 2020), indica que se deben realizar exámenes diferentes examenes, como hemograma completo (permite evaluar los tipos y la cantidad de células en la sangre, entre ellos los globulos blancos, rojos y plaquetas, con el fin de evidenciar si se padece alguna enfermedad), paneles hepáticos (esta prueba evidencia la salud general del hígado), pruebas PCR para detectar COVID-19 y VHS (este examen mide la velocidad de hemosedimentación, es decir detecta el grado de inflamación de los órganos funcionales del organismo, por lo general se incluye en el hemograma completo), pruebas de la función renal y cardíaca, radiografía de tórax, ecocardiograma y electrocardiograma. Realizar la respectiva evaluación clínica permitirá que se aplique el tratamiento adecuado, ya que este síndrome comparte ciertas características con la enfermedad de Kawasaki.

\subsection{Tratamiento}

Actualmente no existe un tratamiento establecido para el SIM, sin embargo algunas Instituciones de salud han visto una mejoría en sus pacientes al usar antiinflamatorios que permitan reducir la inflamación de la parte afectada. Igualmente, el uso de terapias inmunomodulador, que requieren la inmunoglobulina intravenosa, permiten una recuperación rápida. En casos de complicaciones se realiza la resucitación fluida, se efectúa el respectivo soporte respiratorio e inotrópico, y finalmente la oxigenación membranosa extracorpórea, ECMO (Feldstein, et al., 2020).

Los corticosteroides se han usado con frecuencia, los anticoagulantes se usaron de manera variable, la aspirina se indica cuando existe una afección coronaria y al momento de presentar sepsis potencial se le administran antibióticos (CDC, 2020c).

\subsection{Prevención}

Según el Centro para el Control y Prevención de Enfermedades (CDC, 2020d), se deben seguir las siguientes medidas de prevención:
$\checkmark \quad$ Lavarse las manos con frecuencia
$\checkmark$ Evitar las personas enfermas
$\checkmark$ Mantener el distanciamiento
$\checkmark$ Usar mascarillas
$\checkmark$ Limpiar y desinfectar las áreas de contacto
$\checkmark$ Lavar los artículos

\subsection{Enfermedad de Kawasaki y Síndrome Inflamatorio Multisistémico}

La Enfermedad de Kawasaki y Síndrome Inflamatorio Multisistémico comparten diferentes características. En comparación el EK se presenta en los niños cuya edad oscila entre 1 y 2 años (Uehara \& Belay, 2012), el MIS-C presenta afecciones gastrointestinales mucho más prominentes, además, los niveles de plaquetas y linfocitos son más bajos. (Feldstein, et al., 2020)

\subsection{Signos y síntomas del Síndrome Inflamatorio Multisistémico relacionado con COVID-19 que afecta a niños y adolescentes menores de 19 años}

Según Whittaker et al. (2020), en un estudio presentado para describir las características clínicas y de laboratorio de los niños hospitalizados con SIM asociado temporalmente al SARS-CoV-2, se tomaron 58 casos de niños de 8 hospitales de Inglaterra ingresados entre el 23 de marzo y el 16 de mayo del presente año (edad mediana, 9 
años). Los resultados de las pruebas de reacción en cadena de la polimerasa reversa para SARS-CoV-2 fueron positivos en 15 (26\%) pacientes y los resultados de la prueba de IgG del SARS-CoV-2 resultaron positivos en 46 (87\%). En total, 45 (78\%) de 58 niños tuvieron evidencia de infección por COVID-19. Todos los niños presentaron fiebre y síntomas inespecíficos, incluidos vómitos, 31 (53\%) dolor abdominal, 30 (52\%) erupción cutánea y diarrea, 26 (45\%) inyección conjuntival, 29 (50\%) desarrollaron shock y necesitaron fármacos o líquidos de apoyo, 17 (29\%) manifestaron ganglios linfáticos agrandados, 13 (22\%) tuvieron daño renal, 9 (16\%) mostraron manos y pies hinchados, 8 (14\%) las arterias coronarias dilatadas o aneurismas, 6 (10\%) presentaron dolor de garganta. 4 (7\%) desarrollaron ritmos cardíacos anormales, 25 (43\%) requirieron ventilación mecánica y 2 (3\%) necesitaron oxigenación por membrana extracorpórea por disfunción cardíaca grave.

Según Cheung et al. (2020), de acuerdo a su estudio presentado donde se incluyeron 17 pacientes (21 años o menos), hospitalizados en el Irving Medical Center de la Universidad de Columbia / New York-Presbyterian Morgan Stanley Children's Hospital en la ciudad de Nueva York entre el 18 de abril y el 5 de mayo de 2020, los 17 pacientes presentaron fiebre (mediana de 5 días), 14 tuvieron síntomas gastrointestinales, 12 mostraron erupción cutánea, 11 conjuntivitis y 9 tuvieron los labios rojos e hinchados. 3 presentaron niveles bajos de oxígeno y 13 entraron en shock. Catorce manifestaron hallazgos anormales en la radiografía de tórax y 8 cumplieron los criterios de Enfermedad de Kawasaki (EK), y 5 de EK incompleta. Todos los niños mostraron niveles elevados de marcadores inflamatorios, 16 niveles altos de IL-6 en suero, 15 niveles altos de péptido natriurético N-terminal-pro-B-tipo (NT-proBNP), 14 niveles altos de troponina T, 12 niveles reducidos de glóbulos blancos de linfocitos y 11 niveles altos de glóbulos inmaduros. Quince requirieron cuidados intensivos, 10 necesitaron apoyo para controlar la presión arterial baja, ninguno requirió ventilación mecánica, tres presentaron ritmos cardíacos anormales y uno desarrolló aneurisma de tamaño mediano.

\subsection{Tipos de pruebas para el diagnóstico del Síndrome Inflamatorio Multisistémico}

Para evaluar si un paciente presenta el Síndrome Inflamatorio Sistémico, el Centro de Control para Enfermedades recomienda realizar pruebas de laboratorio destinadas a identificar si hay evidencia de inflamación o afectación de órganods multisistémicos $(>=2)$, cardíacos, renales, respiratorios, hematológicos, gastrointestinales, dermatológicos o neurológicos (Weiss et al., 2020), estas pruebas pueden incluir: análisis de sangre, ultrasonido abdominal, y por ende tomar pruebas para detectar la presencia del SARS-CoV-2 mediante RT-PCR, serología o prueba de antígeno; esta debe realizarse antes de administrar IgIV o cualquier tratamiento con anticuerpos exógenos (Belhadjer et al., 2020); otras evaluaciones incluyeron pruebas de referencia cardíacas: ecocardiograma, electrocardiograma, prueba de troponina o enzima cardíaca, péptido natriurético de tipo $B$ (BNP) o NT-proBNP. (CDC, 2020e)

\subsection{Medios de contagio}

El Centro para el Control y Prevención de Enfermedades (CDC, 2020f), recibió informes de 570 casos confirmados de SIM, de los cuales el 99\% (565) dieron positivos a COVID-19, y el 1\% restante ha estado en contacto con alguien que ha dado positivo al SARC CoV-2. La mayoría de los niños desarrollaron el Síndrome Inflamatorio Multisistémico entre 2 y 4 semanas después de haberse infectado por el virus que causa la COVID-19.

\subsection{Número de casos de Síndrome Inflamatorio Multisistémico en el país}

Según el boletín 0022 publicado por el Ministero de Salud Publica de Ecuador (MSP, 2020b) sobre el Síndrome Inflamatorio Multisistémico (SIM) en niños y adolescentes menores de 19 años asociado a COVID-19, en Ecuador se han notificado 128 casos hasta la semana 48, de ellos se le tomaron muestras de laboratorio PCR a 111 niños, de los cuales 8 dieron positivos, 56 negativos y 64 muestras están en espera de resultados, mientras que a 17 no fue posible realizarle la toma de pruebas, por ello se consideran casos probables. Cabe indicar que, del total de los 128 casos, uno fue descartado y falleció con diagnóstico de neumonía viral, no obstante, de los 127 restantes, 7 requirieron hospitalización y 3 fueron trasladados a la Unidad de Cuidado Intensivos (UCI), ya que presentaban un cuadro clínico grave. 
La provincia que presenta un mayor número de casos es Guayas con $53(41,41 \%)$, Pichincha 20 (15,63\%), Los Ríos 18 (14,06 \%), Manabí 14 (10,94\%), Esmeraldas 8 (6,25\%), Tungurahua 5 (3,91 \%), Loja 4 (3,13\%), Azuay y Cañar 2 (3,12 \%), Sucumbíos y Zamora Chinchipe reportan un caso que corresponde al 2,34 \%.

\section{Metodología}

El diseño que se utilizó en esta investigación es de enfoque cualitativo con alcance exploratorio y descriptivo porque permitió plantear diversas alternativas para la recolección de datos, con el fin de buscar respuestas al problema y los objetivos planteados. Debido a que se recopiló información para establecer cuáles son los signos y síntomas del Síndrome Inflamatorio Multisistémico relacionado con COVID-19 que afecta a niños y adolescentes menores de 19 años en el Cantón la Troncal.

Se determinó el medio de contagio del SIM en los niños y adolescentes, el tipo de prueba que se realizó para diagnosticar esta afección y el número de casos reportados.

La investigación se abordó en el ámbito exploratorio, ya que el problema de salud del SIM aún está en proceso de investigación, se manejó un cronograma de actividades para la recopilación de toda la información necesaria.

La información se recolectó por medio del Distrito de Salud 03D03 del Cantón la Troncal, los datos se obtuvieron de forma aleatoria, es decir se recopiló a través de los diferentes registros clínicos, ya sean estos físicos o digitales, acerca de los pacientes que se han atendido durante el período de marzo-agosto de 2021 en este centro de salud.

\section{Resultados y discusión}

\subsection{Casos de SIM presentados en el distrito de salud $03 d 03$ del Cantón la Troncal durante el período de marzo-agosto de 2021}

En el Distrito de Salud 03D03 del Cantón La Troncal durante el período marzo-agosto de 2021, se atendieron a un total de 52 pacientes, niños y adolescentes menores de 19 años de edad (Cuadro 1 y gráfico 1), de los cuales 21 resultaron con probable exposición a COVID-19 (Cuadro 2), por lo que se ingresaron como casos sospechosos de SIM debido a los síntomas presentados.

Cuadro 1

Pacientes atendidos por grupo de edad.

\begin{tabular}{|l|c|c|}
\hline \multicolumn{1}{|c|}{ Edad } & Casos & Porcentaje \\
\hline $0-11$ meses & 10 & $19 \%$ \\
\hline $1-4$ años & 16 & $31 \%$ \\
\hline $5-9$ años & 8 & $15 \%$ \\
\hline $10-14$ años & 14 & $27 \%$ \\
\hline $15-19$ años & 4 & $8 \%$ \\
\hline TOTAL & 52 & $100 \%$ \\
\hline
\end{tabular}

Fuente: Distrito de Salud 03 D03

del Cantón La Troncal.

Del total de los 21 casos ingresados al área de $\mathrm{UCl}$, los grupos de edad más afectados fueron los niños de 1 a 4 años y de 10 a 14 años, con 7 casos respectivamente, sumando entre los dos $66 \%$, seguido por el grupo de edad de 0 a 11 meses, donde se presentaron 4 casos que corresponden al 19\%, de 5 a 9 años se notificaron 3 casos, que pertenecen al 14\%. (Cuadro 2 y gráfico. 2). 
Cuadro 2

Casos sospechosos

de SIM por edad.

\begin{tabular}{|l|c|c|}
\hline \multicolumn{1}{|c|}{ Edad } & Casos & Porcentaje \\
\hline $0-11$ meses & 4 & $19 \%$ \\
\hline $1-4$ años & 7 & $33 \%$ \\
\hline $5-9$ años & 3 & $14 \%$ \\
\hline $10-14$ años & 7 & $33 \%$ \\
\hline $15-19$ años & 0 & $0 \%$ \\
\hline TOTAL & 21 & $100 \%$ \\
\hline
\end{tabular}

Fuente: Distrito de Salud 03D03

del Cantón La Troncal

\subsection{Signos y síntomas de los casos de SIM presentados en el Cantón La Troncal}

Los niños y adolescentes menores de 19 años ingresados en el Distrito de Salud 03D03 del Cantón La Troncal, presentaron los siguientes síntomas asociados a la enfermedad del Síndrome Inflamatorio Multisistémico (Cuadro 3).

Cuadro 3

Signos y síntomas casos

sospechosos de SIM

\begin{tabular}{|l|c|c|}
\hline \multicolumn{1}{|c|}{ Signos y síntomas } & Casos & Porcentaje \\
\hline Fiebre & 21 & $100 \%$ \\
\hline Vómito & 21 & $100 \%$ \\
\hline Dolor abdominal & 21 & $100 \%$ \\
\hline Erupción cutánea & 13 & $62 \%$ \\
\hline Diarrea & 13 & $62 \%$ \\
\hline Conjuntivitis & 21 & $100 \%$ \\
\hline Dolor de garganta & 5 & $24 \%$ \\
\hline Ganglios linfáticos agrandados & 8 & $38 \%$ \\
\hline Inflamación mucocutánea & 7 & $33 \%$ \\
\hline Hipotensión & 9 & $43 \%$ \\
\hline Aneurisma & 8 & $38 \%$ \\
\hline Taquicardia & 3 & $14 \%$ \\
\hline Necesitaron oxigenación & 1 & $5 \%$ \\
\hline
\end{tabular}

Fuente: Distrito de Salud 03D03

del Cantón La Troncal

Los niños y adolescentes, antes de presentar los síntomas, han estado en contacto o expuestos a COVID-19 dentro de 1 a 3 semanas previas, según indicaron los parientes a los médicos. Del total de los 21 casos, los síntomas más recurrentes fueron: fiebre > 3 días, vómito, dolor abdominal y conjuntivitis en el $100 \%$ de los pacientes, 13 presentaron erupción cutánea y diarrea, que corresponden al 62\%, 9 (43\%) mostraron hipotensión, 8 (38\%) tuvieron ganglios linfáticos agrandados y aneurisma, 7 (33\%) manifestaron inflamación mucocutánea, 5 (24 \%) presentaron dolor de garganta, 3 (14 \%) desarrollaron taquicardia y 1 (5\%) necesitaron oxigenación. Cabe indicar que los 21 casos permanecieron en sala Covid restringida. (Gráfico 3). 


\subsection{Medios de contagio}

Durante la recolección de información en el Distrito de Salud 03D03 del Cantón La Troncal los médicos expresaron que los 21 casos registrados manifestaron los síntomas semanas después de estar en contacto con alguna persona infectada por el virus del SARS-CoV-2, ya sea sintomático o asintomático, es decir el medio de contagio recurrente fue la exposición a COVID-19 (Cuadro 4).

\section{Cuadro 4}

Medios de contagio de los

casos sospechosos de SIM

\begin{tabular}{|l|c|c|}
\hline \multicolumn{1}{|c|}{ Medios de Contagio de SIM } & Casos & Porcentaje \\
\hline SARC CoV-2 Positivo & 1 & $5 \%$ \\
\hline Evidencias de Covid-19 & 20 & $95 \%$ \\
\hline TOTAL & 21 & $100 \%$ \\
\hline
\end{tabular}

Fuente: Distrito de Salud 03D03

del Cantón La Troncal

\subsection{Tipos de pruebas que se realizaron para el diagnóstico del SIM}

Los tipos de pruebas que se realizaron a los 21 casos registrados fueron hemograma completo, ultrasonido abdominal, pruebas PCR y pruebas IgG, 7 pacientes necesitaron realizarse un electrocardiograma. De estos 20 pacientes dieron negativo a las pruebas PCR, pero tuvieron evidencias de COVID-19, solo 1 caso dio positivo. (Cuadro 5).

Cuadro 5

Pruebas realizadas a los

casos sospechosos de SIM.

\begin{tabular}{|l|c|c|}
\hline \multicolumn{1}{|c|}{ Pruebas } & Casos & Porcentaje \\
\hline Hemograma & 21 & $100 \%$ \\
\hline Ultrasonido Abdominal & 21 & $100 \%$ \\
\hline Electrocardiograma & 7 & $33 \%$ \\
\hline Prueba PCR & 21 & $100 \%$ \\
\hline Prueba IgG & 21 & $100 \%$ \\
\hline
\end{tabular}

Fuente: Distrito de Salud 03D03

del Cantón La Troncal

\section{Conclusiones}

Según los resultados obtenidos se puede concluir que el Síndrome Unflamatorio Multisistémico que afecta a niños y adolescentes menores de 19 años, está asociado al SARS-CoV-2, ya que los 21 pacientes ingresados en el Distrito de Salud 03D03 del Cantón La Troncal han estado expuestos o cerca de alguna persona que se ha infectado del virus que causa la COVID-19 semanas antes de presentar los síntomas del SIM.

Los síntomas más recurrentes fueron fiebre persistente por más de 3 días, dolor abdominal, vómito, conjuntivitis, diarrea, erupción cutánea, hipotensión, ganglios linfáticos agrandados, aneurisma, inflamación mucocutánea, dolor de garganta, taquicardia y dificultad respiratoria. Cabe destacar que la afección del sistema orgánico y marcadores de laboratorio anormales de inflamación son los criterios que se requieren para incluir a los pacientes dentro del grupo de casos sospechosos de SIM.

Las pruebas destinadas para la evaluación de SIM incluyeron exámenes de laboratorios iniciales en todos los pacientes, los análisis de sangre incluyeron hemograma completo, también se le realizaron ultrasonido 
abdominal, pruebas PCR, pruebas para detectar anticuerpos del SARS-CoV-2, el 33\% de los pacientes requirieron pruebas adicionales que incluían electrocardiograma.

Esta afección se encuentra dentro de los grupos de las enfermedades más desafiantes, tanto para el personal de salud, como para la población, es por ello la necesidad que los profesionales puedan abordar estos casos y concientizar a la población sobre las medidas preventivas a seguir con la finalidad de evitar la infección.

\section{Referencias bibliográficas}

Belhadjer, Z., Méot, M., Bajolle, F., Khraiche, D., Legendre, A., Abakka, S., . . Selegny, M. (2020). Acute heart failure in multisystem inflammatory syndrome in children (MIS-C) in the context of global SARS-CoV-2 pandemic. Circulation, 142(5), 429-436. doi:https://doi.org/10.1161/CIRCULATIONAHA.120.048360

Centers for Disease Control an Prevention - CDC. (2020a). Síndrome Inflamatorio Multisistémico en niños (MISC). Obtenido de https://www.cdc.gov/mis-c/

Centers for Disease Control an Prevention - CDC. (2020b). Para padres: síndrome inflamatorio multisistémico en niños (MIS-C) asociado al COVID-19. Obtenido de https://espanol.cdc.gov/coronavirus/2019-ncov/dailylife-coping/children/mis-c.html

Centers for Disease Control an Prevention - CDC. (2020c). Información para proveedores de atención médica sobre el síndrome inflamatorio multisistémico en niños (MIS-C). Obtenido de https://www.cdc.gov/mis$\mathrm{c} / \mathrm{hcp} /$

Centers for Disease Control an Prevention - CDC. (2020d). Ayuda a detener la propagación de COVID-19 en niños. Obtenido de https://www.cdc.gov/coronavirus/2019-ncov/daily-life-coping/children/protectchildren.html

Centers for Disease Control an Prevention - CDC. (2020e). Definición de caso para MIS-C. Obtenido de https://www.cdc.gov/mis-c/hcp/

Centers for Disease Control an Prevention-CDC. (2020f). Casos notificados por el Departamento de Salud de Síndrome Inflamatorio Multisitémico en niños (MIS-C) en los Estados Unidos. Obtenido de https://www.cdc.gov/mis-c/cases/index.html

Cheung, E., Zachariah, P., Gorelik, M., Boneparth, A., Kernie, S., Orange, J., \& Milner, J. (2020). Síndrome inflamatorio multisistémico relacionado con COVID-19 en niños y adolescentes previamente sanos en la ciudad de Nueva York. JAMA, 294-296. doi:https://doi.org/10.1001/jama.2020.10374

Children, H. (2020). COVID-19 y el síndrome inflamatorio multisistémico en niños. Obtenido de healthychildren.org: https://www.healthychildren.org/spanish/health-issues/conditions/covid19/paginas/covid_inflammatory_condition.aspx

Feldstein, L., Rose, E., Horwitz, S., Collins, J., Newhams, M., \& Son, M. (2020). Multisystem inflammatory syndrome in U.S. children and adolescents. N Engl J Med, 334-346.

doi:https://doi.org/10.1056/NEJMoa2021680

MSP. (2020a). Boletin Epidemiológico SIM 0022. Retrieved from https://www.salud.gob.ec/wpcontent/uploads/2020/12/BOLETIN-SIM-22.pdf 
MSP. (2020b). Alerta Epidemiológica por Síndrome inflamatorio multisistémico (SIM) en niños y adolescentes (menores de 19 años), temporalmente relacionado con COVID-19. Quito - Ecuador. Obtenido de https://www.salud.gob.ec/wpcontent/uploads/2020/06/Alerta_por_si\%CC\%81ndrome_inflamatorio_multisiste\%CC\%81mico_31_05_20 20-MSP.pdf

SOCHINF. (2020). Protocolo síndrome inflamatorio multisistémico en niños, niñas y adolescentes con Sars-Cov-2. Chile. Obtenido de http://www.sochinf.cl/portal/templates/sochinf2008/documentos/2020/Sindromelnflamatorio_Multisiste mico.pdf

Uehara, R., \& Belay, E. (2012). Epidemiología de la enfermedad de Kawasaki en Asia, Europa y Estados Unidos. J Epidemiol. doi:https://10.2188/jea.je20110131

Weiss, S., Peters, M., Alhazzani, W., Agus, M., Flori, H., \& Inwald, D. (2020). Surviving sepsis campaign international guidelines for the management of septic shock and sepsis-associated organ dysfunction in children. Pediatr Critical Care Medicine, 21(2), e52-e106.

doi:https://doi.org/10.1097/PCC.0000000000002198

Whittaker, E., Bamford, A., Kenny, J., Kaforou, M., Jones, C., P, S., \& P, R. (2020). Características clínicas de 58 niños con síndrome inflamatorio multisistémico pediátrico asociado temporalmente con el SARS-CoV-2. JAMA, 259-269. doi:https://doi.org/10.1001/jama.2020.10369

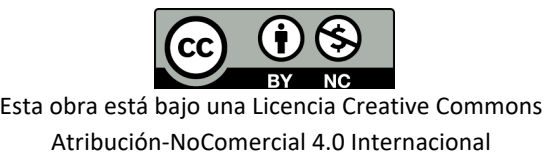

